\title{
Secondary-Electron Electron-Beam-Induced Current Measurements at Lattice Resolution
}

\author{
Matthew Mecklenburg $^{1 *}$, William A. Hubbard ${ }^{2,3}$, Jared J. Lodico ${ }^{2,3}$, and B. C. Regan ${ }^{2,3}$ \\ 1. Core Center of Excellence in Nano Imaging, University of Southern California, Los Angeles, USA. \\ 2. Department of Physics and Astronomy, University of California, Los Angeles, USA. \\ 3. California NanoSystems Institute, University of California, Los Angeles, USA. \\ * Corresponding author: matthew.mecklenburg@usc.edu
}

Using an aberration-corrected scanning transmission electron microscope (STEM), we demonstrate lattice resolution imaging in gold/palladium nanoparticles via electron beam-induced current (EBIC) imaging of secondary electron (SE) emission. As the focused STEM beam is rastered across the electron transparent sample, SEs are emitted from above and below the sample with a yield of a few percent [1]. The holes left behind are collected as a current by a transimpedance amplifier, and the resulting voltage signal is associated with the beam position to form a SE emission EBIC, or SEEBIC, image [1]. SEEBIC provides efficient and quantitative SE detection, is sensitive to all emitted electrons regardless of emission geometry (e.g. top vs. bottom surface, detector position), and does not require additional modification of the electron microscope (beyond use of an electrical biasing holder).

To demonstrate lattice resolution with STEM EBIC imaging, we use a commercially available grating replica magnification calibration sample. Because of the low secondary yield, large primary beam currents are necessary to give an EBIC image with a satisfactory signal to noise ratio. We use an aberration-corrected STEM (NCEM's TEAM 1.0) with $~ 300 \mathrm{pA}$ of beam current, and an accelerating voltage of $300 \mathrm{kV}$. We are able to achieve a lattice resolution of roughly $0.24 \mathrm{~nm}$ (see orange arrow, Fig. 1). In the Fig. 1 SEEBIC image, both the nanoparticle and the carbon film produce similar contrast, as SEEBIC is sensitive to electron emission from both the nanoparticle and the film on which it sits. Thus we are able to resolve lattice even with the added "background" of the signal from the carbon film. In regions of carbon film alone, the HAADF image gives very little signal, while the SEEBIC shows details of the carbon film surface. The ability to simultaneously resolve multiple layers of elements with drastically different atomic numbers could prove useful for niche imaging purposes, including biological samples.

Aberration-corrected SE imaging has been previously demonstrated using an off-sample detector [2]. Aside from the aforementioned advantages of SEEBIC over remote SE detection, SEEBIC has the additional advantage of providing differential contrast depending on where current is measured [1]. While imaging a device, for example, SEEBIC can be collected from individual electrodes, providing information about electronic structure, such as conductance changes, which are invisible to both STEM and SE imaging. SEEBIC has also demonstrated promise at providing a measure of work function [1]. The highly localized beam-induced hole currents result from secondary electron emission from not only the conduction band, but also core states [3]. Correlating atomic-scale electronic features with the atoms themselves can drastically advance understanding of device physics at the most basic level. 
References:

[1] W A Hubbard et al., Physical Review Applied, 10 (2018), 044066.

[2] Y Zhu et al., Nature Materials, 8 (2009), p. 808-812.

[3] E J Sternglass, Physical Review, 108 (1957) p. 1.

[4] This work was supported by National Science Foundation (NSF) Science and Technology Center (STC) award DMR-1548924 (STROBE), and by NSF award DMR-1611036. Work at the Molecular Foundry was supported by the Office of Science, Office of Basic Energy Sciences, of the U.S. Department of Energy under Contract No. DE-AC02-05CH11231.

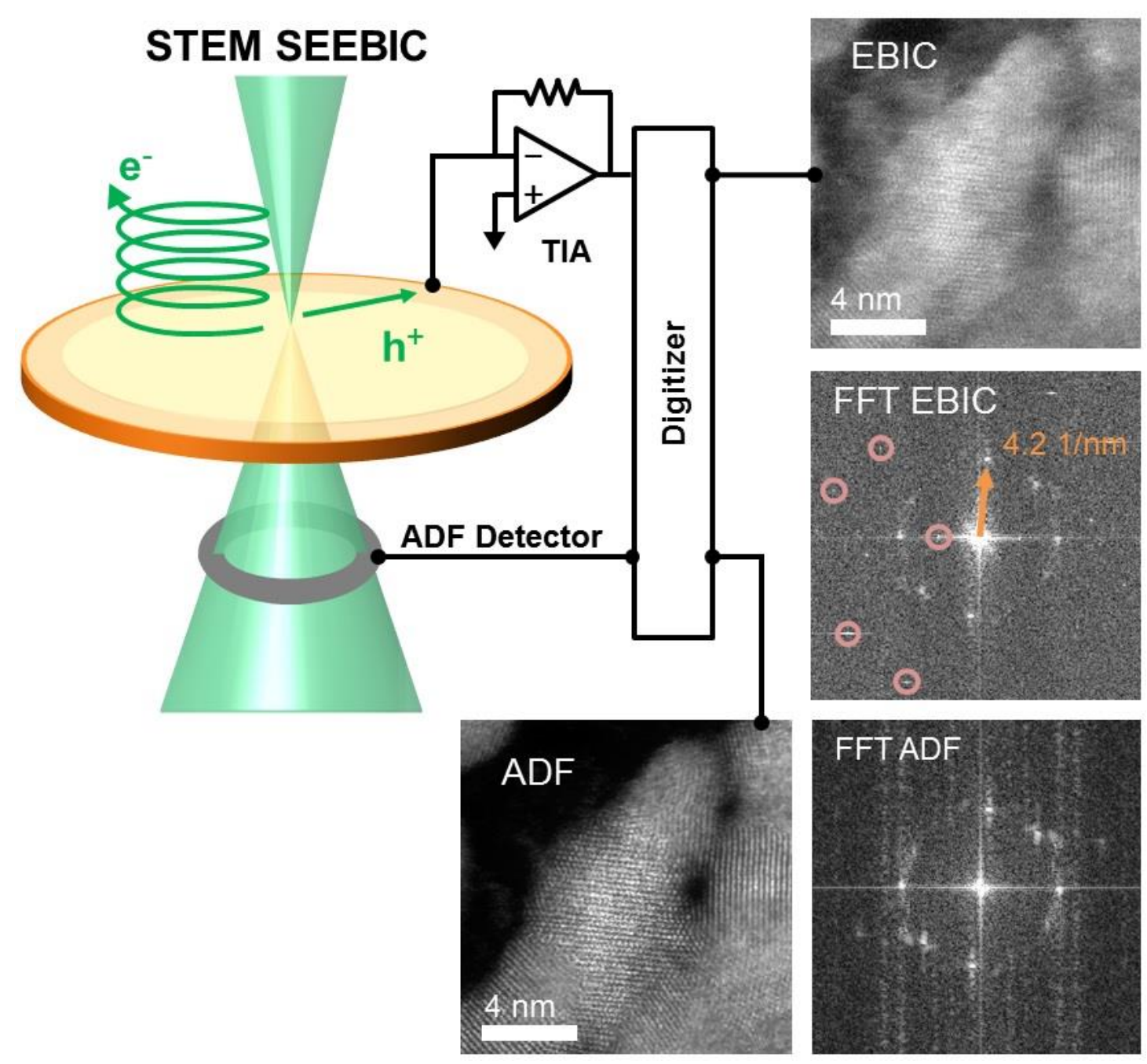

Figure 1. Cartoon showing secondary electron emission, hole creation, and hole detection through the transimpedance amplifier (TIA), along with detection of the scattered electrons via the annular dark field (ADF) detector. Representative, simultaneously acquired ADF and EBIC images are shown, along with the corresponding fast Fourier transforms (FFTs). The similarity of the FFTs demonstrates that both imaging techniques have detected the same crystal lattice. A small amount of $60 \mathrm{~Hz}$ noise (the 5 circled peaks and their symmetric partners) has been filtered from the EBIC image via the EBIC FFT. 